\title{
Bioactive specific biomaterials: Present and future
}

\author{
Jacqueline Jozefonvicz and Marcel Jozefowicz \\ Laboratoire de Recherches sur les Macromolécules, CNRS URA 502, \\ Avenue J.B. Clement, 93430 Villetaneuse, France
}

\begin{abstract}
Bioactive biomaterials made of synthetic or artificial polymers substituted with specific chemical functional groups carried by the macromolecular chain are designed to develop specific interactions with living systems. These soluble or insoluble polymers are derived from polystyrene and dextran.

Such functional polymers may be endowed with anticoagulant heparin-like properties and, as a consequence, possess low thrombogenicity when they are in contact with flowing blood. Other functional polymers have been tailored specifically to interact with components of the immune system. Other polymers, in contact with cells can affect both cell growth and cell biological functions or only cell biological activity without necessarily undergoing change in all characteristics.

Derived from the above concepts, it is possible to demonstrate that a random statistical distribution of chemical groups along the macromolecular backbone correlates with the biological properties of these polymers.
\end{abstract}

\section{INTRODUCTION}

When a polymeric material is exposed to a biological environment, there is a natural tendency to induce different reactions such as blood coagulation, complement activation and cell interactions. The biologic species which are involved in this process are proteins and cells. The first event is generally non-specific protein adsorption on biomaterials, as a consequence of different regions of the molecule of protein which are available to interact with the numerous local binding sites on the polymers depending on the heterogeneities of the biomaterial. This protein adsorption is highly irreproducible depending on the processing of the polymer which, in turn, controls the distribution of the local heterogeneities of the binding sites. The successive displacement of plasma proteins adsorbed to artificial surfaces, fixed by the concentration of protein and by the rate of transport, is called the Vroman effect. This process is responsible for the activation of the blood coagulation process which occurs when blood contacts polymeric biomaterials (ref. 1). Activation of the complement system followed by both cellular and humoral immune responses may also result from the early stages of the protein adsorption process (ref. 2). In fact, both protein adsorption onto biomaterials and cell interaction with biomaterials are spatial arrangements of molecules. With cells, the molecules which are involved are extracellular matrix proteoglycans, sulfate proteoglycans synthesized by the cell, receptors and growth factors. Moreover, these multiple binding possibilities control the healing process into porous polymer networks and the formation of fibrous capsules around polymeric prostheses.

The specific properties of bioactive polymers result from the non-random distribution on the polymeric surfaces of specific chemical groups. Depending on the chemical nature of the functional groups and their relative proportions in the final product, these bioactive polymers are endowed with binding sites for proteins, polypeptides and proteoglycans which will range from non-specific to highly specific.

In order to investigate the structure-function relationships between living system and substituted polymers, we hypothesized that a random distribution of given or particular chemical groups on the polymer could confer specific interactions with some proteins or cells. In the following we shall present some results based on this hypothesis. 


\section{ANTICOAGULANT ACTIVITY OF SUBSTITUTED DEXTRANS}

Heparin-like soluble and biodegradable dextrans have been obtained by random substitution of the polymer with carboxylic and benzylamide sulfonate groups (Fig. 1).<smiles>COC[C@H]1O[C@@H](CI)[C@H](OC)[C@H](O)[C@H]1OCC(=O)O</smiles>

D

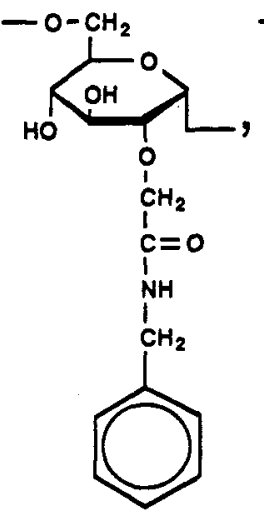

B

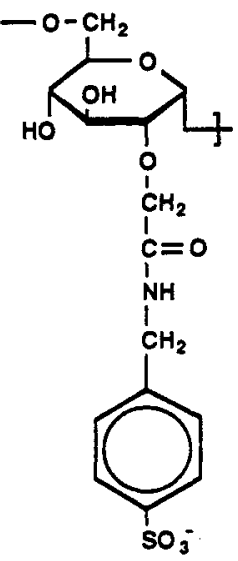

$\mathbf{S}$

Fig. 1. Structure of derivatized dextrans or crosslinked derivatized dextrans.

These materials exhibit an anticoagulant activity strongly dependent upon the respective percentages of the monomer units bearing each substituent as indicated in Fig. 2 (ref. 3).

Results show that there is no significant anticoagulant activity until the average percentage of the dextran units bearing carboxylic groups becomes greater than $40 \%$. In the absence of benzylamide sulfonate groups, carboxylic substituted units do not exhibit activity. Also the anticoagulant activity increases with benzylamide sulfonate content. The composition of the samples refers to global distribution along the polymer backbone. Thus, the data suggest that specific structural

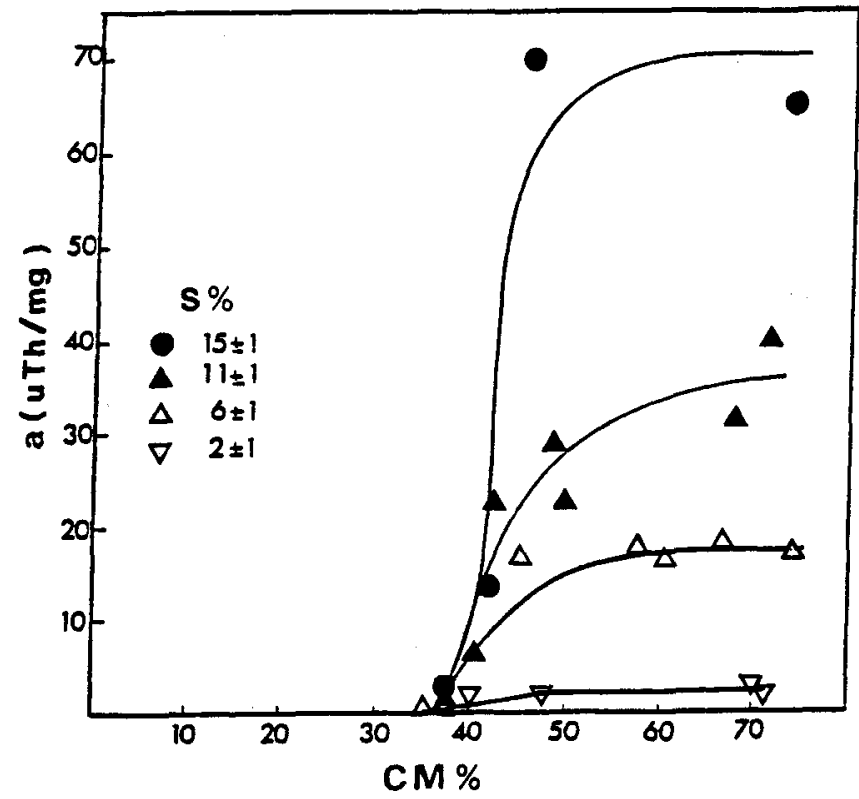

Fig. 2 Anticoagulant activity of dextran derivatives expressed in units of thrombin inhibited per $\mathrm{mg}$ of dextran derivative (a $u T \mathrm{Th} / \mathrm{mg}$ ) versus the percentage of carboxymethyl dextran units (CM \%) for different samples bearing various dextran units substituted with benzylamide sulfonate groups (S \%) (ref. 3). 
determinants regulate anticoagulant activity of derivatized dextrans. We postulate that a specific sequence can be responsible for binding derivatized dextrans to antithrombin which is the principal plasma protein involved in anticoagulant action via heparin or heparin-like functional dextrans. It is possible that such a sequence (e.g. the pentasaccharide sequence of heparin obtained by synthesis containing a 3-0-sulfated glucosamine unit (ref. 4)) contains a domain that binds to antithrombin and possesses a high specific activity against procoagulant proteases. The probability of obtaining numerous macromolecules of derivatized dextran containing the specific sequence then depends on the conditions of substitution of glycosyl rings of native dextran. Possible correlations between theoretical substitution of glycosyl rings and experimental results are currently being investigated.

\section{BIOACTIVE POLYMERS AND ANTIBODIES}

Antibodies are specific proteins, immunoglobin (lg) molecules which are produced by plasma cells when B cells are exposed to a particular antigen. Such antigens may be isolated molecules (toxins, proteins) or may be molecules at the surface of an invading foreign cell or foreign biomaterial. These immunoglobins share similar biologic functions but can have varied specificities for antigens. They are composed of polypeptide chains divided into an Fab (antigen-binding) fragment and an Fc (crystallizable) fragment which present constant regions and variable regions. The specificity of an antibody against an antigen is correlated with the chemical nature of the variable regions.

Biospecific functional polymers will interact specifically with the protein through its Fab fragment if the nature and proportions of the chemical groups randomly grafted onto the macromolecular backbone polymer are able to create sequences that possess a high affinity for specific sites in the Fab region. This occurs with some crosslinked polystyrene derivatives which are antigen-like.

One example is provided by insoluble polymers in which tyrosine sulfamide, aspartic sulfamide and sulfonate groups are present on the crosslinked polystyrene beads. The derivatized polymers mimic epitopes of the Factor VIII structure recognized by anti-Factor VIII antibodies. These antibodies, arising in 8 to $16 \%$ of multitransfused hemophiliacs, neutralized the procoagulant activity of Factor VIII via amino acid residues, aspartyl (Asp ${ }^{1663}$ ) and tyrosyl (Tyr ${ }^{1664}$ ) (ref. 5). The goal of the study was to selectively adsorb anti-Factor VIII antibodies from plasma with derivatized resins. Table 1 shows that the percentage of adsorbed anti-Factor VIII antibodies is the highest and the percentage of total adsorbed $\operatorname{lgG}$ is the lowest when 1.2 milliequivalents of tyrosine residues are linked to one gram of sulfonated polystyrene beads.

TABLE 1. Adsorption respectively of anti-Factor VIII antibodies (AFVIII \%) and Immunoglobin $\mathrm{G}$ (IgG \%) on different derivatized beads. The milliequivalents of active ligand linked per gram of beads (meq/g) is given for tyrosyl (-Tyr) and Aspartyl (-Asp).

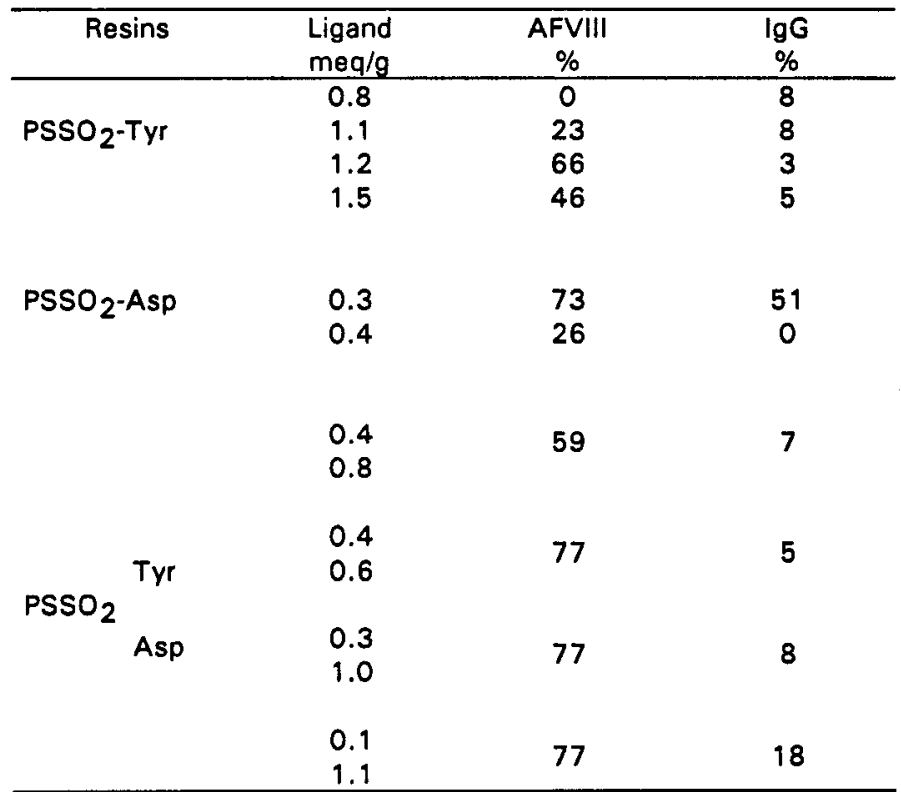


The co-substituted resins where tyrosine and aspartic residues are linked to beads are able also to remove a high titer of anti-Factor VIII provided they are substituted with a low level of aspartyl residues (0.6 milliequivalent/g) (ref. 6).

A second example is related to phosphorylated polystyrene derivatives (ref. 7). These insoluble functional polymers have been developed to interact via their antigen binding sites with systemic lupus erythematous (SLE) anti-DNA antibodies (ref. 8). Polystyrene units bearing phosphate groups :

$$
\text { PS- } \mathrm{CH}_{2}-\mathrm{CH}_{2}-\mathrm{O}-\mathrm{PI}-(\mathrm{OH})_{2}
$$

are randomly distributed along the macromolecular backbone and the substituted polystyrene mimics the epitope of the DNA structure recognized by these specific antibodies which form stable complexe with either single or double stranded DNA. The phosphomonoesters of hydroxylated polystyrene resins adsorb anti-DNA antibodies. The affinity constants for antibody adsorption, evaluated from the adsorption isotherms assuming that they obey a Langmuir type law, were found to be on the order of $10^{8} \mathrm{M}^{-1}$, whereas the binding constant for the total IgG is about $10^{5} \mathrm{M}^{-1}$.

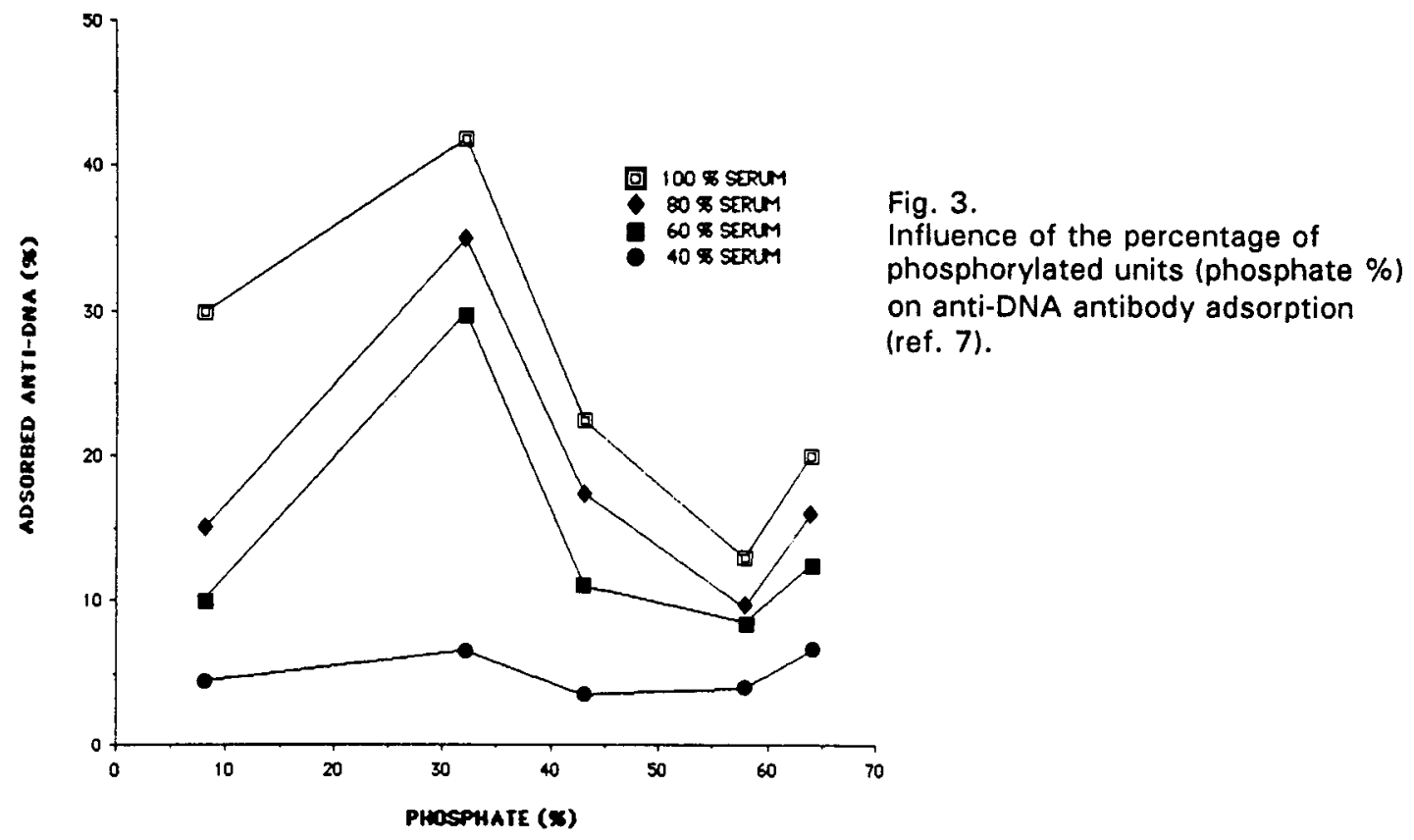

Fig. 3 shows that the percentage of adsorbed anti-DNA antibodies depends on the degree of substitution of phosphorylated beads (ref. 9). The adsorption is maximum when $30 \%$ of the hydroxylated monomer units are phosphorylated and becomes minimum when $60 \%$ of such units are present on the beads. The anti-DNA antibodies bind to the beads via their antigen binding regions with high association constants. These beads can be used for SLE biological diagnosis for the detection of autoimmune antibodies in the sera of patients. Based upon the above data, we believe that a random distribution of phosphorylated groups on the hydroxylated polystyrene could confer high specific interactions with antibodies when numerous macromolecules on the derivatized beads contain the specific domain that binds to the antibodies.

\section{BIOACTIVE POLYMERS AND CELLS}

Heparin glycosaminoglycan with anionic carboxylic and sulfate groups exerts complex effects on smooth muscle cell (SMC) or endothelial cell growth. In some cases these effects depend on the growth factors present in culture media. The family of derivatized dextrans (Fig. 1) which possess four types of glycosyl units statistically distributed along the macromolecular backbone (nonsubstituted glycosyl units (D) and glycosyl units respectively substituted with carboxymethyl (CM) benzylamide (B) and benzylamide sulfonate (S) groups) exhibit an effect on the regulation of cell growth. 
These functionalized dextrans are obtained by random substitution of native dextrans by suitable chemical groups (ref. 3). Some of them with different contents of $C M, B$ and $S$ units have a high capacity to inhibit SMC, in vitro. This capacity is generally of the same order of magnitude as that for heparin. The data indicate that benzylamide groups are required to increase anti-proliferative activity and that the materials showing the greatest anticoagulant activity are not the most active in inhibiting cell growth. These data reveal that anti-proliferative activity clearly requires structural determinants distinct from those endowing anticoagulant activity (ref. 10). Other results relate to the in vitro stimulation of human umbilical vein endothelial cells (HUVEC) by this family of derivatized dextrans. The effect on mitogenesis of HUVEC in the absence of serum leads to the conclusion that exogenous growth factors are not involved in the proliferative capacity of these materials (ref. 11). It appears that benzylamide sulfonate groups play an important role in the stimulation of HUVEC. Besides, the mitogenic capacity of derivatized dextrans strongly depends upon the respective ratios of the functional groups. Preliminary structural analysis of these macromolecules was made in order to determine the critical sequence which constitutes the cellbinding domain. We also showed that distinct populations of these CMDBS macromolecules are able to bind with high affinity respectively to CML and to HUVEC. In addition, our results suggest that these biologically active CMDBS polymers have an effect on the regulation of cell growth which strongly depends on the nature of the cells.

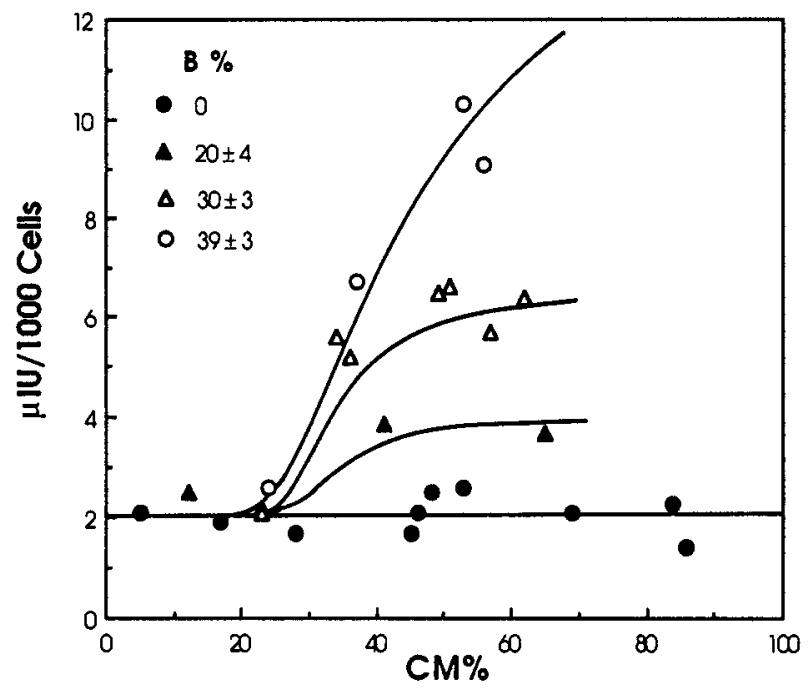

Fig. 4.

Insulin secretion by RINm5F cells expressed in microunits of insulin per 1000 cells versus the percent age of carboxymethyl dextran units (CM \%) for different crosslinked dextran beads bearing various benzylamide groups (B \%) (ref. 13).

Insoluble derivatized polymers should also be able to interact with cell membrane receptors such as insulin-receptors entrapped in the phospholipid membrane of the cells (ref. 12). Direct or mediated interactions may modify the biological activity of the cells. Crosslinked derivatized dextran beads interact with an insulin secreting cell line, RINm5F, derived from a radiation induced rat insulinoma. These interactions are not mediated by any binding protein. The data show that the insulin secreted by the cells controls insulin secretion when RINm5F cells are cultured on crosslinked derivatized dextran beads obtained by random substitution of native crosslinked dextran beads with, respectively, carboxylic and benzylamide groups (see Fig. 1 ; without $S$ units) (ref. 13). As a result, an insulin stimulating effect is observed; this insulin secretion stimulating effect was shown to be reversible and dependent on the percentage of the glycosyl units bearing carboxyl groups (CM \%) (Fig. 4). Moreover the data show that there is no stimulating effect until the average percentage of these latter units becomes greater than $25 \%$. In the absence of benzylamide groups, beads derivatized with carboxyl groups did not stimulate RINm5F cells, while the stimulating effect increases with increasing content of benzylamide glycosyl units. The effect is five-fold greater than the insulin secretion effect induced by soluble secretary agents. It should be noted that these experiments show once again the important role of the specific benzylamide group as was observed in the stimulation of HUVEC by soluble derivatized dextrans. Moreover, two soluble derivatized dextrans with the same composition as the two corresponding crosslinked derivatized dextrans have absolutely no stimulating effect on the insulin secretion of RINm5F cells. This fact demonstrates that although soluble and insoluble functional dextrans probably interact specifically with the same cell membrane receptor, this interaction results in a different cell response in the two cases because of the lack of internalization of the ligand-receptor complex when the biospecific biomaterial is insoluble. 


\section{REFERENCES}

1. E.F. Leonard and L. Vroman, \. Biomater. Sci. Polymer Edn 3, 95-107 (1991).

2. M.D. Kazatchkine and M.P. Carreno, Biomaterials $9,30-35$ (1988).

3. M. Mauzac and J. Jozefonvicz, Biomaterials 5 , $301-304$ (1984).

4. J. Choay, M. Petitou, J.C. Lormeau, P. Sinay, B. Casu and G. Gatti, Biochem. Biophys. Res. Comm. 116, 492-499 (1983).

5. M. Shima, C.A. Fulcher, S. de Graaf Mahoney, R.A. Houghten and T.S. Zimmerman, J. Biol. Chem. 263, 10198-10203 (1988).

6. M. Algiman, Y. Sultan, C. Boisson and J. Jozefonvicz, XIX Int. Congress of the World Federation of Hemophilia, Wahington DC, August 14-19, 1990, abstract.

7. D. Letourneur, C. Douzon and M. Jozefowicz, J. Polym. Sci. Polym. Chem. Ed. 29, 1367-1377 (1191).

8. D. Letourneur, Thesis, Paris-North University (1988).

9. D. Letourneur and $M$. Jozefowicz, Biomaterials (in press).

10. T. Avramoglou and J. Jozefonvicz, J. Biomater. Sci. Polymer Edn. (in press).

11. D. Letourneur, J. Champion, F. Slaoui and J. Jozefonvicz, In vitro (submitted).

12. N. Aubert, G. Reach, H. Serne and M. Jozefowicz, J. Biomed. Mat. Res. 21 585-601 (1987).

13. N. Oturan, Thesis, Paris-North University (1990). 